\title{
A hundred years after the first article, a recollec- tion: Cabot ring
}

\author{
Neslihan Erdem ${ }^{1}$, İlhami Berber ${ }^{2}$, İsmet Aydoğdu ${ }^{3}$, and Alper Sevinç ${ }^{4}$
}

${ }^{1}$ Department of Internal Medicine, Celal Bayar University Faculty of Medicine, Manisa; ${ }^{2}$ Department of Hematology, Inonu University Faculty of Medicine, Malatya; ${ }^{3}$ Department of Hematology, Celal Bayar University Faculty of Medicine, Manisa; ${ }^{4}$ Department of Medical Oncology, Gaziantep University Faculty of Medicine, Gaziantep, Turkey

Received: October 22, 2014 Revised : November 6, 2014 Accepted: January 25, 2015

\section{Correspondence to}

Neslihan Erdem, M.D.

Tel: +90-55-5729-8822

Fax: +90-23-6233-8040

E-mail: neslihnerdem@gmail.com
We share our experience with Cabot ring and report that it is seen not only in megaloblastic anemia and lead poisoning, but also in other hematological diseases such as myelofibrosis and chronic myeloid leukemia.

Richard Cabot described Cabot ring in the blood smears of anemic patients as ring-shaped structures. The physiological mechanism of Cabot ring is unclear, it is reported to involve arginine-rich histones and non-hemoglobin iron; it is also seen in megaloblastic anemia, lead poisoning, homozygous thalassemia, and splenectomy. Reviewing our image records, we have seen Cabot ring in many hematological diseases, including myelofibrosis (Fig. 1) and chronic myeloid leukemia (Fig. 2). In our patients, Cabot ring was seen mostly in basophilic red cells, and in blood smears with polychromatic red cells, as described by Cabot. Based on the basophilic staining and common characteristic of the diseases in which this structure is seen, we postulate that it is seen in diseases with stressed bone marrow. Cabot ring is a nuclear remnant, which supports the hypothesis. Although 100 years have passed, we still do not know the clinical significance of Cabot ring. However, as Cabot stated, “...the safest rule in scientific work is to describe everything new that we come across, whether we see any significance in it or not." Therefore, we aimed to report the other diseases in which we have seen Cabot ring 100 years after the first report.

\section{Conflict of interest}

No potential conflict of interest relevant to this article was reported.
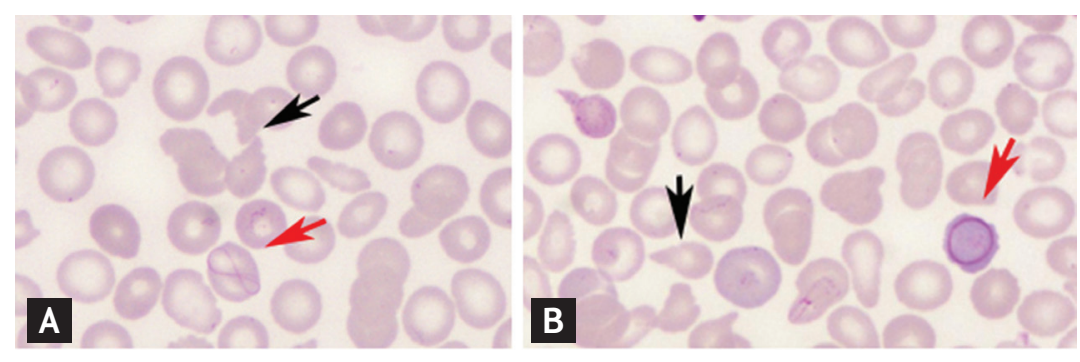

Figure 1. (A, B) Cabot ring in myelofibrosis, red arrows show Cabot ring, black arrows show dacrocytes.

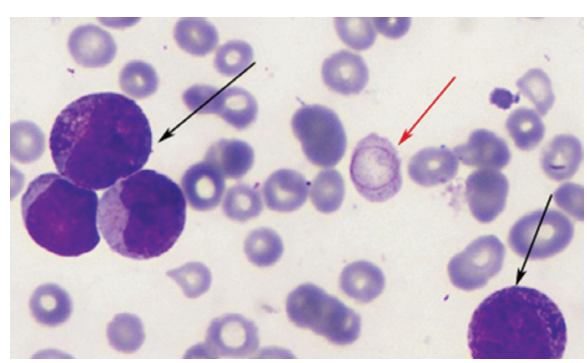

Figure 2. Chronic myeloid leukemia, red arrow shows Cabot ring, black arrows show increased leukocytes and different types of granulocytes. 